\title{
SUB-CHRONIC MPTP-INDUCED PARKINSONISM: SELECTIVE SENSITIVITY OF THE BALB/C MICE AND THE PROPHYLACTIC ROLES OF NIGELLA SATIVA OIL
}

\author{
Royhaan Folarin ${ }^{1}$, Akinola Olonade ${ }^{1}$, Praise Ogunkunle ${ }^{1}$, Philemon Shallie ${ }^{1}$, Thomas \\ Adenowo $^{1}$, Muinat Adeyanju $^{2}$, Paul Folarin ${ }^{1}$, Tarilayefa Amangele ${ }^{1}$, \\ ${ }^{1}$ Neurophytotherapy Unit, Department of Anatomy, Olabisi Onabanjo University, Sagamu, Nigeria \\ ${ }^{2}$ Department of Biochemistry, Olabisi Onabanjo University, Sagamu, Nigeria
}

*Corresponding Author: Royhaan Folarin

Neurophytotherapy Unit (NPTU), Department of Anatomy,

Olabisi Onabanjo University, Sagamu, Nigeria

royhaan.folarin@oouagoiwoye.edu.ng+2347032064419

\begin{abstract}
Parkinsonism is a degenerative neurological syndrome characterised by dopamine deficiency in the basal ganglia. Despite ages of research, there is still lack of holistic and side-effect-free therapy for the disease. Nigella sativa is a multi-potent plant known for its historical and scientifically proven therapeutic potentials in the brain and other body organs. Despite the validity debates on the use of BALB/c mice strains in the modelling of Parkinsonism using MPTP, this study re-appraised the sensitivity versus resistance of the BALB/c mice strain to MPTP and further investigated the possible neuro-thrapeutic role of Nigella sativa oil (NSO) in the Parkinsonic endophenotypes elicited by MPTP in the BALB/c strain. Body weights, relative brain weights, striatal dopamine, striatal neuron density and recognition memory were studied in thirty-two (32) male albino mice, weighing between $18 \mathrm{~g}-25 \mathrm{~g}$. They were divided equally into Control (administered with normal feed for 5 days), MPTP (administered with 18mg/kg MPTP i.p for 5 days), NS (administered with 1ml/kgbw NSO p.o. for 5 days), and NS+MPTP (administered with $1 \mathrm{ml} / \mathrm{kgbw}$ NSO p.o. followed by $18 \mathrm{mg} / \mathrm{kg}$ MPTP i.p for 5 days). Recognition memory was assayed through Novel Object Recognition test (NORT), and the animals were weighed and euthanised 24 hours after last administration. The brains were excised and the striatum assayed neurochemically for dopamine and illustrated histologically for neuronal density using the $H \& E$ stain. Parkinsonic traits such as mild tremor, significant down-regulation of dopamine and striatal neurons $(\mathrm{p}<0.05)$ were recorded in the BALB/c mice administered with MPTP only, confirming MPTP-sensitivity for these features. However, significant increase $(\mathrm{p}<0.05)$ in appetite, body weight, brain-body weight ratio, and recognition memory was also recorded in the MPTP-administered mice, though Nigella sativa was significantly prophylactic against the negative Parkinsonic features, and 'moderative' of the up-regulations induced by MPTP. While this suggests selective MPTP sensitivity and resistance in BALB/c strains, this study recommends the investigation of possible beneficial potentials of MPTP as observed. The research was conducted in conformance with the Animal Research Ethics Committee (AREC) guidelines of the Olabisi Onabanjo University.
\end{abstract}

Keywords: Nigella sativa, MPTP, BALB/c, Parkinsonism, Selectivity, Striatum 


\section{Introduction}

Parkinson's disease (PD) is a degenerative disorder of the central nervous system that mainly affects the motor system, \{National Institute for Neurological Disorders and Stroke (NINDS, 2019)\}. It is characterised by progressive neuronal degeneration which predominantly affects the dopaminergic neurons in the nigrostriatal system and several other regions of the brain (Roberts et al., 1994). The exact cause of Parkinson's disease (PD) is unknown, but epidemiological studies suggest an association with environmental toxins. Early in the disease, the most obvious are shaking, rigidity, slowness of movement, and difficulty with walking; thinking and behavioural problems may also occur (NINDS, 2019). Other symptoms include sensory, sleep, and emotional problems. The main motor symptoms are collectively called "parkinsonism", or a "parkinsonian syndrome" (D. R. Williams \& Litvan, 2013). The main obstacle to developing neuroprotective therapies is a limited understanding of the key molecular events that provokes neurodegeneration (G. V. Williams \& Goldman-Rakic, 1995).

The striatum is a principal component of the basal ganglia (a group of nuclei that have a variety of functions but are best known for their role in facilitating voluntary movement, thought to be involved in diverse aspects of cognition and behaviour. However, while movement, reward, and motivation are the most studied of functions associated with the striatum, they are by no means the extent of them (Dingman, 2015).

Nigella sativa (also referred to as Habbatus-sawdaa - Black seed) is one of the most widely used medicinal plants across the world (Ahmad et al., 2013), with a long history of use across ancient Egyptian, Indian (Unani), Greek (Ayurvedic), Roman and Islamic cultures (Al-Naqeep, Al-Zubairi, Ismail, Amom, \& Esa, 2011). It is an important therapeutic agent in the Tibbun-nabawiyy system of Islamic medicine, regarded as a "remedy for all diseases except death" (Al-Bukhari, 1997). Nigella sativa is processed into capsules as dietary supplements, while its oil is employed in cooking and topical (external) application in first aid and home therapy. It also forms a major constituent of body creams, roll-ons, massage creams, shampoos and bathing soaps. With therapeutic effects on multiple body systems in humans and animals (Assi et al., 2016), Nigella sativa has also proven useful in the treatment of psychiatric disorders (Akram Randhawa \& Ahmed Alenazi, 2016). However, due to the paucity of information on its impacts on MPTP-induced Parkinsonism, particularly on striatal neurons, this research was aimed at filling this knowledge gap.

In order to replicate the disorder for better understanding and research, the use of BALB/c mice in MPTP modelling of Parkinsonism has been a subject of debate. While some researchers like Sedelis et al. (2000) simply regarded the BALB/c as less susceptible to MPTP than the C57BL/6 due to neurochemical, behavioural and histological data; others like Filipov, Norwood, \& Sistrunk, (2009) 
added age dependence to the sensitivity, such that aging is regarded as a factor that reduces the MPTP resistance in both BALB/c and C57BL/6. For example, Sedelis et al. (2000) reported that recovery of $\mathrm{BALB} / \mathrm{c}$ mice to the control levels after administration of MPTP was faster than in C57BL/6; and that nest building was also not delayed in the BALB/c mice unlike in the C57BL/6. MPTP treatment, however, did not lead to any significant loss of TH-immunoreactive cells in the ventral tegmental area (VTA) of the BALB/c mice.

As variations however abound amidst these findings, this study aimed at appraising the above reports while investigating the possible neuro-therapeutic role of Nigella sativa oil in the Parkinsonic endophenotypes, through the analyses of brain-body weights ratio, body weights, striatal dopamine level, striatal neuron density, and recognition memory. This is without precluding the need to replicate the same motive using the more sensitive C57BL/6 mice strain.

\section{Materials and Methods}

\subsection{Acquisition of research materials}

Sixty (60) mls of Nigella sativa oil was procured from Hemani ${ }^{\circledR}$ International, Pakistan. Similarly, 1-methyl-4-phenyl-1-2-3-6-tetrahydropyridine (MPTP) was procured from Medchem Express, New Jersey, USA. The thirty-two (32) adult male albino mice used for this study, with weight ranging from $18 \mathrm{~g}$ to $25 \mathrm{~g}$ were acquired from the Animal Lab of the Faculty of Basic Medical sciences, Sagamu. Grower's mash feed, manufactured by Animal care ${ }^{\circledR}$ Limited was procured at one of the company's authorised depot in Ikenne, Ogun state, Nigeria. The animals were housed in their respective well ventilated cages, improvised from transparent plastic boxes, and with drinking and feeding troughs constructed with plastic bottles suspended within the cages. The animals were maintained under controlled temperature and humidity in the Experimental Animal Holding Facility of the Basic Medical Sciences, Sagamu. Ethical clearance no. BS/18/VII/23-010 was obtained for this research from the Anatomical Research Ethics Committee (AREC), Olabisi Onabanjo University, Sagamu, Nigeria.

\subsection{Experimental Design and Dosing}

The animals were randomly divided into four (4) groups, as shown in table 1 below, and allowed to acclimatise for one week.

Table 1 Showing administered substances and respective dosages

\begin{tabular}{|r|l|l|l|l|c|}
\hline S/N & Group & $\begin{array}{c}\text { No. of } \\
\text { mice }\end{array}$ & $\begin{array}{c}\text { Administered } \\
\text { substance }\end{array}$ & Dosage & $\begin{array}{c}\text { Duration of } \\
\text { administration }\end{array}$ \\
\hline 1. & Control & 12 & Normal Saline & $0.05 \mathrm{ml} / \mathrm{kg}$ & 5 days \\
\hline 2. & MPTP & 12 & MPTP only & $18 \mathrm{mg} / \mathrm{kg}$ & 5 days \\
\hline
\end{tabular}




\begin{tabular}{|r|l|l|l|l|l|}
\hline 3. & NS & 12 & $\begin{array}{l}\text { Nigella sativa oil } 1 \mathrm{ml} / \mathrm{kg} \\
\text { only }\end{array}$ & \multicolumn{1}{|c|}{5 days } \\
\hline 4. & NS+MPTP & 12 & $\begin{array}{l}\text { Nigella sativa oil } 1 \mathrm{ml} / \mathrm{kg} \mathrm{\&} \\
\text { and MPTP } \\
\mathrm{mg} / \mathrm{kg} \\
\text { respectively }\end{array}$ & $\begin{array}{l}\text { Nigella sativa oil for } 5 \text { days } \\
\text { followed by subsequent } \\
\text { MPTP for the next } 5 \text { days }\end{array}$ \\
\hline
\end{tabular}

Following respective administrations, the mice were placed on video surveillance for one hour each, to observe their behavioural responses to the respective regimens, especially the MPTP.

\subsection{Measurement of Body Weight}

The body weights of the animals were measured the commencement and end of the study using a Kero ${ }^{\circledR}$ sensitive weighing balance in order to check for weight gain and/or loss across the groups.

\subsection{Novel Object Recognition (NOR) Test}

The Novel Object Recognition (NOR) test was performed for each group of mice, 24 hours after the final administration. The test involved the habituation, training and testing phases. During the habituation phase, each mouse was introduced into an open field maze $(38 \mathrm{~cm} \times 38 \mathrm{~cm} \mathrm{x} 38 \mathrm{~cm})$ to get habituated for 5mins. During the training phase, each mouse was placed into the open field maze for another 5 minutes, this time containing objects A and B, both of which are similar in shape, colour, size and height. They were placed diagonally within the maze and equidistant from each other. During the testing phase however, the mice were again introduced into the open field maze, but this time, with object $B$ already replaced with a novel object $C$ at the same location. This phase also lasted for 5 minutes. The test was tracked with a video camera, and the time spent by each animal in exploring the novel object $C$ was recorded against the time spent exploring the familiar object $A$ and the discrimination index was determined as a percentage of the novel object exploration time over the total time spent exploring both objects.

\subsection{Sacrifice}

Following administration and neurobehavioral assays, the animals were sacrificed. Each group of animals was randomly divided into two categories, and were sacrificed either by cervical dislocation, for immediate excision and homogenization of the striatum in $0.1 \mathrm{M}$ phosphate buffered saline (PBS) for further neurotransmitter assay, or euthanized with $0.1 \mathrm{ml}$ ketamine hydrochloride (i.p.), followed by transcardiac perfusion with $0.9 \%$ normal saline for two minutes, and then $0.1 \mathrm{M}$ phosphate buffered saline (PBS). The brain tissues were subsequently fixed in formal calcium solution for twenty-four hours. The perfused brains were taken through the routine $\mathrm{H} \& \mathrm{E}$ processing. 


\subsection{Brain Weights}

The respective weights of the excised brains were measured, and the relative brain weights (RBW) of the animals across the groups was calculated using the formula:

$$
\text { RBW }=(\text { Brain Weight } / \text { Body Weight }) \times 100
$$

\subsection{Dopamine assay}

The homogenised tissues were centrifuged at $5000 \mathrm{rpm}$ for 5 minutes, and a liquid chromatography-tandem mass spectroscopy (LC-MS/MS) method with protein precipitation was employed to assay the levels of dopamine (DA) in the striatum.

\subsection{Statistical analysis}

Data acquired were expressed as mean \pm standard error of mean, and were analyzed with oneway analysis of variance (ANOVA) using the GraphPad Prism (version 7.0) software. Statistical significance was set at $\mathrm{p}<0.05$. 


\section{Results}

The following are the recorded results at the end of the research.

\subsection{Physical observations}

Following the first administration session, the MPTP mice expressed mild tremor and reduced locomotion. Their food and water consumption rates also increased appreciably, as observed after the first day, even though this was not actively measured. The feed consumption rate for the NS group was however lower than the control, even though, all other groups apart from the MPTP maintained their normal levels of activity after their respective administrations throughout the study. The activity levels of the NS+MPTP mice however increased slightly upon commencement of MPTP administration. This however did not reach the level of the MPTP mice.

However, an idiopathic scoliosis (IS) developed in in one of the MPTP mice on the fourth day of administration. The animal was thereafter isolated for observation and possible recuperation. However, the animal never recovered until it died three days afterwards.

\subsection{Body Weight Changes}

The following were weight changes recorded during the research

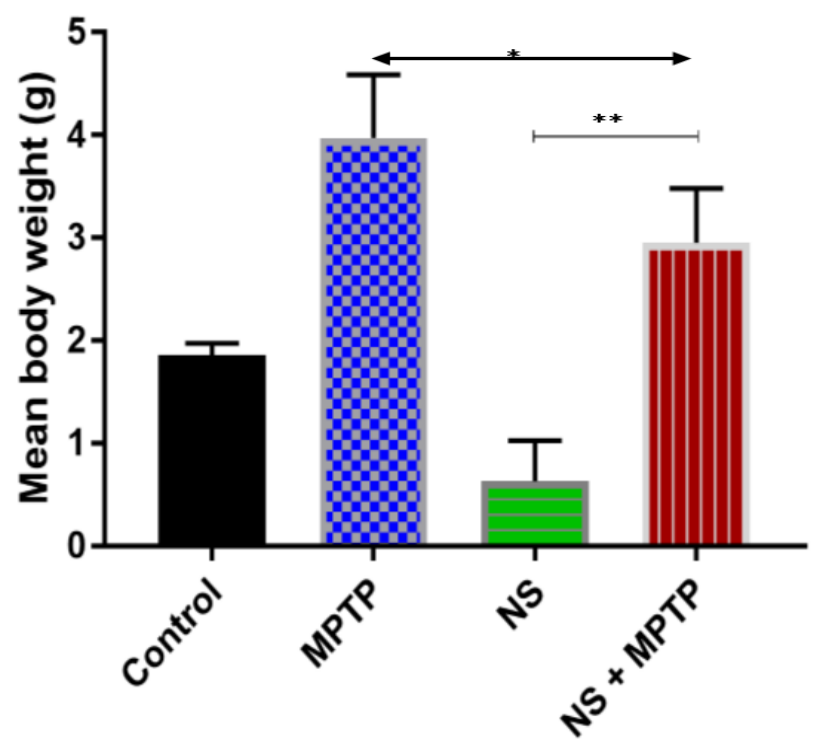

Figure 1 Graph showing the mean body weight difference $(\mathrm{g})$ of the animals in different groups ( $*$ and ${ }^{* *}$ indicate increasing levels of significance at $\mathrm{p}<0.05$ )

Figure 1 shows the mean difference in body weights (final weight - initial weight) of the animals. The MPTP mice had the highest and thus significantly higher mean body weight than mice of all other groups. Also, NS group had the least MBW when compared with the Control group while NS+MPTP group had a significantly lesser MBW than the MPTP group but higher than the NS group. 


\subsection{Relative Brain Weight}

The following are the brain weight results obtained from the study

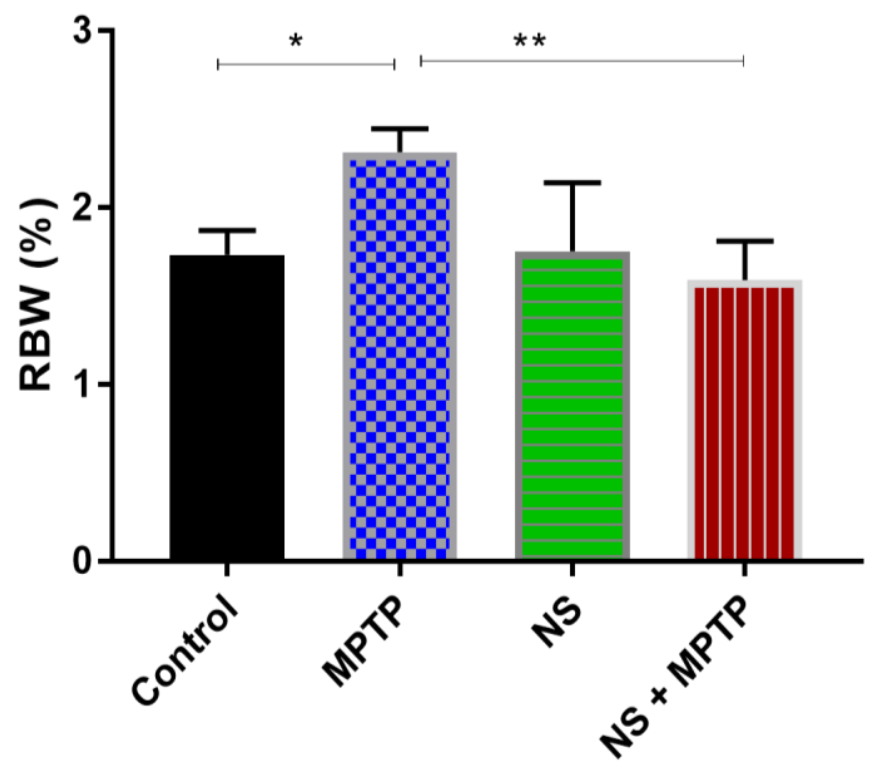

Figure 2 Graph Illustrating Relative Brain Weights (RBW) in Experimental Mice (* and ${ }^{* *}$ indicate increasing levels of significance at $\mathrm{p}<0.05$ )

The relative brain weights of NS+MPTP group was found to be lower than all the groups while that of MPTP group was the highest. Also, RBW of NS group was slightly higher than found in the Control and NS+MPTP groups.

\subsection{Novel Object Recognition Test}

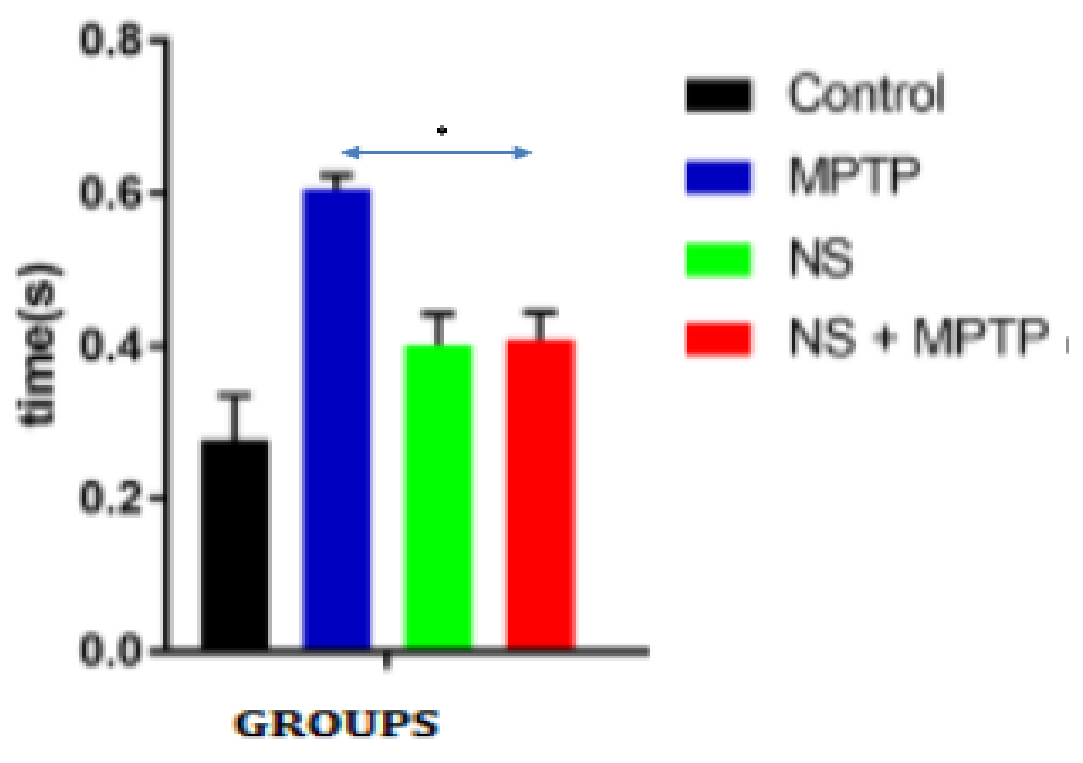

Figure 3 Graph showing Novel Object Recognition in Experimental Mice ${ }^{*}$ and ${ }^{* *}$ indicate increasing levels of significance at $\mathrm{p}<0.05$ ) 
Figure 3 above shows a significantly higher novel exploration time for the MPTP group than all other groups. The NS+MPTP mice however had a similar exploration time with the NS mice, while both had higher exploration time than the control mice.

\subsection{Dopamine Assay}

The following are the results obtained from the dopamine assay

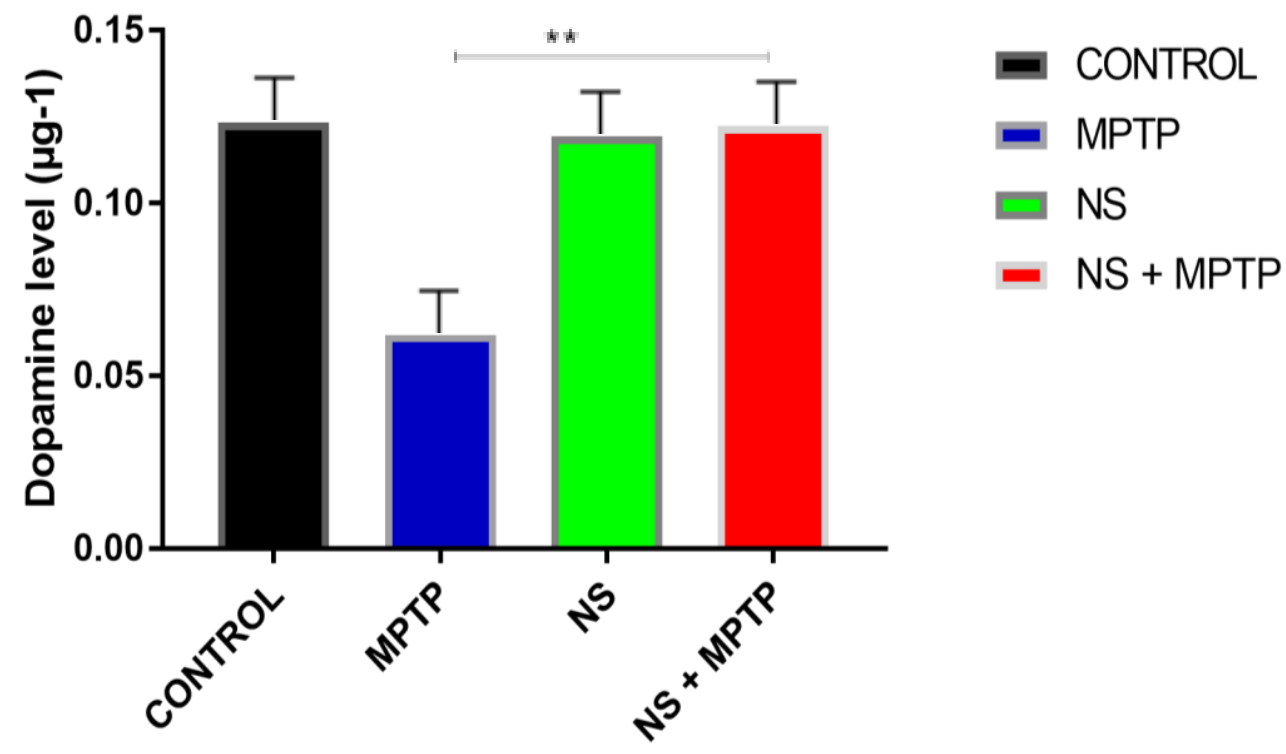

Figure 4 Graph illustrating Dopamine level in Striatum of Experimental Mice ${ }^{*}$ and ${ }^{* *}$ indicate increasing levels of significance at $\mathrm{p}<0.05$ )

Figure 4 above shows no significant difference in the dopamine levels expressed by the striatum of the Control, NS and NS+MPTP mice. The MPTP group expressed the lowest striatal dopamine level, with a significantly lower level than all other groups. The NS+MPTP mice therefore had a higher dopamine level than the parkinsonic mice. 


\subsection{Hematoxylin and Eosin Stains}

Shown below are photomicrographic results obtained using the H\&E stain
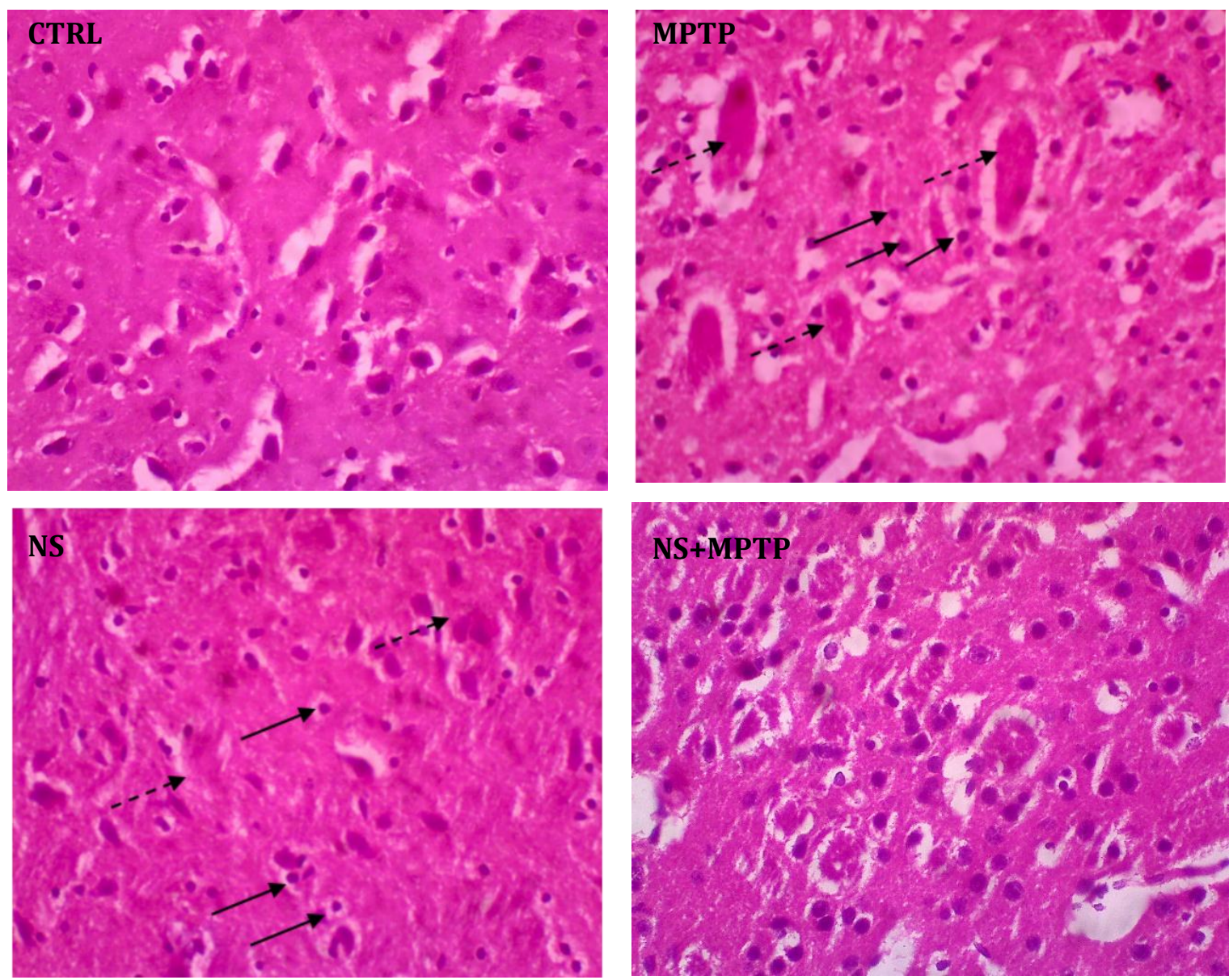

Neuronal Density

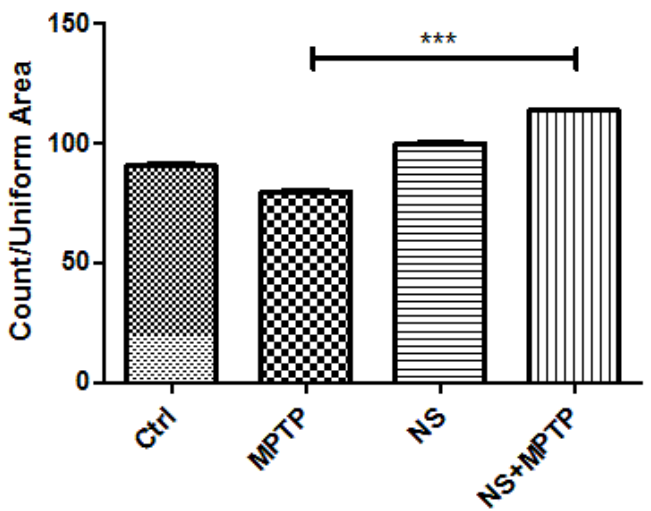

Figure 5 Photomicrographs of the Striatum stained with H \& E stains across all mice groups. Magnification $=(\mathrm{X400})$. Full arrows indicate striatal neurons, while dashed arrows indicate nigrostriatal pathway bundles

Figure 5 above shows the striatal histology across the experimental mice, with striatal neurons and bundles of nigrostriatal pathways indicated in full and dashed arrows respectively. The neuronal density in the MPTP mice was the least while the NS+MPTP mice expressed significantly higher striatal neurons than the MPTP, control and the NS mice. 


\section{Discussion}

As earlier reported, motor symptoms such as mild tremor and reduced locomotion, were observed in the MPTP mice following the first administration. This validates earlier reporters including Aarsland, Londos, \& Ballard (2009), Jellinger, (1998) and Meredith \& Rademacher (2011), in which rigidity, slowness of movement, postural instability, and freezing were reported in MPTP-administered humans, non-human primates, cats, rabbits, and some rodents strains. Furthermore, this study further shows that BALB/c mice are also sensitive to MPTP with respect to this Parkinsonic feature. This is in contrast to utter categorisations of the BALB/c mice as MPTP-resistant (Meredith \& Rademacher, 2011).

For the basis for these sensitivity differences to MPTP 'between' mice strains is yet to be well understood despite several explanations proposed, more research is also be required to further determine the cause of the varied sensitivity 'within' same strains of species. However, these parkinsonic traits were absent in the mice pre-administered with Nigella sativa (NS+MPTP), indicating the prophylactic potential of Nigella sativa in preventing Parkinsonism.

Idiopathic scoliosis (IS) has been described as a multifactorial disease involving many intrinsic factors such as genetics, imbalance of muscle structures, abnormal growth of vertebral bodies, asymmetrical growth of the neurocentral cartilage, length discrepancy between spine and spinal cord, abnormal platelet calmodulin, and abnormality in melatonin metabolism (Banala, Vemuri, Penkulinti, Av, \& Gpv, 2018; Machida, 2018). The idiopathic scoliosis observed in one of the MPTP mice may however be an epiphenomenon rather than being consequential to the MPTP administered, as has been said of many hypothesised factors (Machida, 2018).

Human PD patients have been reported to experience low body weight, a phenotype hypithesised to be predisposed by many factors dysphagia, chewing difficulty, impaired hand-mouth coordination, and hyposmia (Bachmann \& Trenkwalder, 2006; Ma et al., 2018). Similar body weight trend has also been reported of non-human primates (Porras, Li, \& Bezard, 2012). Despite this trend however, no significant weight loss was recorded in C57BL/6 mice by Sundström, Fredriksson, \& Archer (1990) during 4 weeks after MPTP exposure.

An obvious contrast to the above trend was the finding of the current study, in which the MPTP mice had the highest mean body weight increase compared with all other groups. While this may be explained by the increased food and water consumption rates observed in the mice (even though this was not actively measured), it is a further validation of the report in which $32.9 \%$ of PD patients studied by Cersosimo et al. (2018) showed increase in their body weights. This study thus shows MPTP as increasing MBW in BALB/c mice. The improved appetite and the consequent 
body weight gain also raises the question about the tendency of MPTP or perhaps some of its active constituents to improve diet and body weights, as found in the BALB/c mice here studied.

However, the weight gain in the NS mice was significantly lower, and in fact the least when compared to other groups. This is similarly explained by the feed consumption rate which was lower in the NS group than observed in all other groups. Reduced weight gain as recorded in this study, is a phenomenon that has been previously characterised to Nigella sativa exposure, similar to the finding of Bano, Wajeeh, Baig, Naz, \& Akhtar (2009) and Le et al. (2004).

The weight limiting effect of Nigella sativa was thus seen on the NS+MPTP mice which had a significantly lesser MBW than the MPTP group but higher than the NS group. Nigella sativa was thus able to limit the weight gain in this group.

Relative brain weight (RBW), also known as brain-to-body weight ratio, is a hypothetical estimate of the intelligence of an animal, as it is believed to coincide much better with the observed cognitive abilities than absolute brain size (Cairó, 2011). In this study, the highest RBW was recorded in the MPTP mice. This indicates MPTP's ability to effect increase in RBW possibly through a progression of the brain weight along with the body weights of the MPTP animals as discussed above. The NS+MPTP mice however recorded significantly lower RBW than the MPTP mice, indicating the impact of NS pre-exposure in keeping the RBW at par with the level recorded in the NS mice.

Novel object recognition test (NORT) is a measure of recognition memory which harnesses the innate tendency of mice to explore novelty of their environment (SBFNL(b), 2019). The MPTP mice showed the highest exploration time with the novel object in the assay, when compared with other groups, thus accruing the highest recognition memory to the MPTP mice. This finding is inconsistent with the previous reports in which mild cognitive impairment and dementia are established as pathophysiological features of Parkinson's disease at the early and later stages respectively (Weil, Costantini, \& Schrag, 2018). Recognition memory deficit particularly has been further documented by some studies in patients with Parkinson's disease (Chiaravalloti et al., 2014). The improved recognition memory found in this study may be explained by the higher brain-body weight ratio found in the MPTP mice, since the later, as earlier explained is hypothesised as a measure of brain function.

MPTP is a known neurotoxin with neurodegenerative potentials in human and animals (Langston, 2017). However, the memory and brain weight findings in the BALB/c mice administered with MPTP raises questions about possible advantageous impacts of this neurotoxin on some brain functions, even if such impacts are species or strain dependent. 
Conversely, mice administered with Nigella sativa oil only (the NS group) showed a lesser recognition memory than the MPTP mice, and a higher (though insignificant) recognition memory than the control mice through their exploration time with the novel object. The exploration time of the NS+MPTP mice was significantly lower than recorded in the MPTP mice but similar with the NS mice. This may suggest the impact of Nigella sativa in moderating the recognition memory in the pre-treated mice.

This study thus found the MPTP mice to express the lowest striatal dopamine, in validation of previous studies which characterised Parkinsonism with deficiency of dopamine and dopaminergic neurons in the midbrain, especially in the striatum (Scherman et al., 1989; Triarhou, 2013). The dopamine levels in the NS and control group however were similar and significantly higher than in the MPTP mice. This may therefore explain the higher dopamine level recorded in the NS+MPTP mice than the parkinsonic mice. This indicates the prophylactic role of Nigella sativa in Parkinsonism.

The neuronal densities of the striatum across the experimental mice groups were detailed in figure 5. The neuronal density in the MPTP mice was the least, in validation of reports by Ross et al., (2004) where the mean neuronal densities in all striatal quadrants were significantly lower in the PD group compared with the other groups. This thus confirms the depletion of striatal neurons in BALB/c strains of MPTP mice models of Parkinsonism, in slight contrast to its being referred to as MPTP-resistant by Meredith \& Rademacher (2011). Therefore, MPTP, when administered alone, conferred the least striatal neuronal density to the mice, in keeping with the known phenotypes of Parkinsonism.

However, when pre-treated with Nigella sativa oil, administering MPTP yielded a significantly higher striatal neuronal density than in mice administered with MPTP only and Nigella sativa oil only. While the level in the mice administered with Nigella sativa only was already higher significantly than the MPTP mice, it is not understood why co-administration would yield a level higher than in the NS only group. This is a recommended subject for further research. The fact, however, that the NS+MPTP mice expressed more striatal neurons than the MPTP mice, corroborates the prophylactic potential of Nigella sativa against Parkinsonic endophenotypes as earlier described with other data above.

Strains of mice are known to differ in their response to MPTP with respect to the degree of striatal DA depletion, amount of loss of midbrain DA neurons, and behavioural deficits. However, while the hallmark of sensitivity and resistance in the substantia nigra -pars compacta (SNpc) was put at ">50\% SNpc neuron loss" and "<25\% SNpc loss" respectively (Meredith \& Rademacher, 2011), the neuronal loss recorded in the BALB/c MPTP mice was only significant statistically, but not up 
to $20 \%$ of the control's neuronal density. A ">50\% loss" was however recorded in the dopamine assays in the MPTP mice.

\section{Conclusion}

The validity of BALB/c mice in the modelling of Parkinsonism using MPTP has been a subject of debate. This study examined the same research design, but with the aim of investigating the role that may be played by Nigella sativa, a potential neuro-therapeutic agent. MPTP was validated with some known Parkinsonic features such as tremor, down-regulation of dopamine and reduced density of striatal neurons in the BALB/c mice. The BALB/c mice may thus be considered MPTP-sensitive as regards these Parkinsonic features. But MPTP also increased the appetite, body weight, brain-body weight ratio, and recognition memory in the MPTP-administered mice. Could this be a beneficial role of MPTP? Nigella sativa was found to ameliorate the negative Parkinsonic features earlier mentioned, as induced by MPTP administration, and also moderated the up-regulations effected by MPTP.

While oral administration of Nigella sativa oil is proven to remain a potential therapeutic agent in the treatment of neurological disorders, as shown in its prophylactic roles against striatal dopamine and neuronal down-regulation in MPTP-administered mice, this study establishes the need to demystify the possible health-supportive tendencies of MPTP as shown in the non-treated mice, while identifying its responsible constituents for such positive effects as found in this study.

\section{Conflict of Interest}

The authors declare no conflict of interest.

\section{Acknowledgements}

The lead author further shows immense gratitude to Prof. Marina Bentivoglio and Pof. Evelyne Sernagor of the IBRO/ISN Writing papers workshop, 2019, for their guidance.

\section{References}

Aarsland, D., Londos, E., \& Ballard, C. (2009). Parkinson's disease dementia and dementia with Lewy bodies: Different aspects of one entity. International Psychogeriatrics, 21(02), 216. https://doi.org/10.1017/S1041610208008612

Ahmad, A., Husain, A., Mujeeb, M., Khan, S. A., Najmi, A. K., Siddique, N. A., ... Anwar, F. (2013). A review on therapeutic potential of Nigella sativa: A miracle herb. Asian Pacific Journal of 
Tropical Biomedicine, 3(5), 337-352. https://doi.org/doi:10.1016/S22211691(13)60075-1

Akram Randhawa, M., \& Ahmed Alenazi, S. (2016). Neuropsychiatric Effects of Nigella sativa (Black Seed) - A Review. Alternative \& Integrative Medicine, 05(01). https://doi.org/10.4172/2327-5162.1000209

Al-Bukhari, M. (1997). The Translation of the Meanings of Sahih Al-Bukhari. Riyadh: Dar-us-Salam Publications.

Al-Naqeep, G., Al-Zubairi, A. S., Ismail, M., Amom, Z. H., \& Esa, N. M. (2011). Antiartherogenic Potential of Nigella sativa Seeds and Oil in Diet-Induced Hypercholesterolaemia in Rabbits. Evidence-Based Complementary and Alternative Medicine, 213628.

Assi, M. A., Mohd Noor, M. H., Bacheck, N. F., AHMAD, H., HARON, A. W., MOHD YUSOFF, M. S., \& RAJION, M. A. (2016). The Various Effects of Nigella Sativa on Multiple Body Systems in Human and Animals. Pertanika Journal of Scholarly Research Reviews, 2(3), 1-19.

Bachmann, C. G., \& Trenkwalder, C. (2006). Body weight in patients with Parkinson's disease. Movement Disorders: Official Journal of the Movement Disorder Society, 21(11), 18241830. https://doi.org/10.1002/mds.21068

Banala, R. R., Vemuri, S. K., Penkulinti, M., Av, G. R., \& Gpv, S. (2018). Development of Novel Animal Model for Studying Scoliosis Using a Noninvasive Method and Its Validation through Gene-Expression Analysis. Asian Spine Journal, 13(1), 126-134. https://doi.org/10.31616/asj.2018.0108

Bano, F., Wajeeh, M., Baig, N., Naz, H., \& Akhtar, N. (2009). Antiobesity, antihyperlipidemic and hypoglycemic effects of the aqueous extract of Nigella Sativa seeds (Kalongi). 42(4), 136140.

Cairó, 0. (2011). External Measures of Cognition. Frontiers in Human Neuroscience, 5. https://doi.org/10.3389/fnhum.2011.00108

Cersosimo, M. G., Raina, G. B., Pellene, L. A., Micheli, F. E., Calandra, C. R., \& Maiola, R. (2018). Weight Loss in Parkinson's Disease: The Relationship with Motor Symptoms and Disease Progression [Research article]. https://doi.org/10.1155/2018/9642524

Chiaravalloti, N. D., Ibarretxe-Bilbao, N., DeLuca, J., Rusu, O., Pena, J., García-Gorostiaga, I., \& Ojeda, N. (2014). The source of the memory impairment in Parkinson's disease: Acquisition versus retrieval. Movement Disorders: Official Journal of the Movement Disorder Society, 29(6), 765-771. https://doi.org/10.1002/mds.25842

Dingman, M. (2015, February 15). Know your brain: Striatum. Retrieved 14 July 2019, from Neuroscientifically Challenged website: https://neuroscientificallychallenged.com/blog/know-your-brain-striatum

Filipov, N. M., Norwood, A. B., \& Sistrunk, S. C. (2009). Strain-specific sensitivity to MPTP of C57BL/6 and BALB/c mice is age dependent. Neuroreport, 20(7), 713-717. https://doi.org/10.1097/WNR.0b013e32832aa95b

Jellinger, K. A. (1998). Neuropathology of movement disorders. Neurosurgery Clinics of North America, 9(2), 237-262.

Langston, J. W. (2017). The MPTP Story. Journal of Parkinson's Disease, 7(Suppl 1), S11-S19. https://doi.org/10.3233/JPD-179006

Le, P. M., Benhaddou-Andaloussi, A., Elimadi, A., Settaf, A., Cherrah, Y., \& Haddad, P. S. (2004). The petroleum ether extract of Nigella sativa exerts lipid-lowering and insulin-sensitizing actions in the rat. Journal of Ethnopharmacology, 94(2-3), 251-259. https://doi.org/10.1016/j.jep.2004.04.030 
Ma, K., Xiong, N., Shen, Y., Han, C., Liu, L., Zhang, G., ... Wang, T. (2018). Weight Loss and Malnutrition in Patients with Parkinson's Disease: Current Knowledge and Future $\begin{array}{llll}\text { Prospects. Frontiers in Aging Neuroscience, } & 10 .\end{array}$ https://doi.org/10.3389/fnagi.2018.00001

Machida, M. (2018). Neurological Research in Idiopathic Scoliosis. In M. Machida, S. L. Weinstein, \& J. Dubousset (Eds.), Pathogenesis of Idiopathic Scoliosis (pp. 157-188). https://doi.org/10.1007/978-4-431-56541-3_7

Meredith, G. E., \& Rademacher, D. J. (2011). MPTP Mouse Models of Parkinson's Disease: An Update. Journal of Parkinson's Disease, 1(1), 19-33. https://doi.org/10.3233/JPD-201111023

NINDS. (2019). Parkinson's Disease Information Page. Retrieved 14 July 2019, from National Institute of Neurological Disorders and Stroke (NINDS) website: https://www.ninds.nih.gov/Disorders/All-Disorders/Parkinsons-Disease-InformationPage\#disorders-r1

Porras, G., Li, Q., \& Bezard, E. (2012). Modeling Parkinson's Disease in Primates: The MPTP Model. Cold Spring Harbor Perspectives in Medicine, 2(3). https://doi.org/10.1101/cshperspect.a009308

Roberts, A. C., De Salvia, M. A., Wilkinson, L. S., Collins, P., Muir, J. L., Everitt, B. J., \& Robbins, T. W. (1994). 6-Hydroxydopamine lesions of the prefrontal cortex in monkeys enhance performance on an analog of the Wisconsin Card Sort Test: Possible interactions with subcortical dopamine. The Journal of Neuroscience: The Official Journal of the Society for Neuroscience, 14(5 Pt 1), 2531-2544.

Ross, G. W., Petrovitch, H., Abbott, R. D., Nelson, J., Markesbery, W., Davis, D., ... White, L. R. (2004). Parkinsonian signs and substantia nigra neuron density in decendents elders without PD. Annals of Neurology, 56(4), 532-539. https://doi.org/10.1002/ana.20226

SBFNL(b). (2019). 2-Object Novel Object Recognition. Retrieved 5 January 2019, from Stanford Behavioral and Functional Neuroscience Laboratory website: https://med.stanford.edu/sbfnl/services/bm/lm/bml-novel.html

Scherman, D., Desnos, C., Darchen, F., Pollak, P., Javoy-Agid, F., \& Agid, Y. (1989). Striatal dopamine deficiency in Parkinson's disease: Role of aging. Annals of Neurology, 26(4), 551-557. https://doi.org/10.1002/ana.410260409

Sedelis, M., Hofele, K., Auburger, G. W., Morgan, S., Huston, J. P., \& Schwarting, R. K. W. (2000). MPTP Susceptibility in the Mouse: Behavioral, Neurochemical, and Histological Analysis of Gender and Strain Differences. Behavior Genetics, 30(3), 171-182. https://doi.org/10.1023/A:1001958023096

Sundström, E., Fredriksson, A., \& Archer, T. (1990). Chronic neurochemical and behavioral changes in MPTP-lesioned C57BL/6 mice: A model for Parkinson's disease. Brain Research, 528(2), 181-188. https://doi.org/10.1016/0006-8993(90)91656-2

Triarhou, L. C. (2013). Dopamine and Parkinson's Disease. Retrieved from https://www.ncbi.nlm.nih.gov/books/NBK6271/

Weil, R. S., Costantini, A. A., \& Schrag, A. E. (2018). Mild Cognitive Impairment in Parkinson's Disease-What Is It? Current Neurology and Neuroscience Reports, 18(4). https://doi.org/10.1007/s11910-018-0823-9

Williams, D. R., \& Litvan, I. (2013). Parkinsonian Syndromes. Continuum : Lifelong Learning in Neurology, $\quad 19(5 \quad$ Movement $\quad$ Disorders $), \quad 1189-1212$. https://doi.org/10.1212/01.CON.0000436152.24038.e0 
Williams, G. V., \& Goldman-Rakic, P. S. (1995). Modulation of memory fields by dopamine D1 receptors in prefrontal cortex. Nature, 376(6541), 572-575. https://doi.org/10.1038/376572a0 